\title{
Rejuveinix Mitigates Sepsis-Associated Oxidative Stress in the Brain: Clinical Impact Potential in COVID-19 and Nervous System Disorders
}

\section{Fatih M Uckun}

Reven Pharmaceuticals

Mehmet Tuzcu

Firat University: Firat Universitesi

Marcus Gitterle

Santa Rosa Hospital: Hospital Santa Rosa

Michael Volk

Reven Pharmaceuticals

Kazim Sahin ( $\nabla$ nsahinkm@yahoo.com )

Firat Universitesi Veteriner Fakultesi

\section{Research Article}

Keywords: Sepsis, Acute lung injury, Multi-organ dysfunction, Cytokine release syndrome (CRS), COVID-19

Posted Date: May 13th, 2021

DOI: https://doi.org/10.21203/rs.3.rs-501838/v1

License: (c) (i) This work is licensed under a Creative Commons Attribution 4.0 International License. Read Full License 


\section{Abstract}

Here, we demonstrate that our anti-sepsis drug candidate Rejuveinix (RJX), at a dose level that is $>10$ times lower than its maximum tolerated dose (MTD) for human subjects, improves the survival outcome in the LPS-GaIN model of sepsis and multi-organ failure. One hundred (100) percent (\%) of untreated control mice remained alive throughout the experiment. By comparison, $100 \%$ of LPS-GalN injected mice died at a median of 4.6 hours. In contrast to the invariably fatal treatment outcome of vehicle-treated control mice, $40 \%$ of mice treated with RJX $(n=25)$ remained alive with a 2.4 -fold longer median time survival time of 10.9 hours (Log-rank $X^{2}=20.60, P<0.0001$ ). Notably, RJX increased the tissue levels of antioxidant enzymes SOD, CAT, and GSH-Px, and it reduced oxidative stress in the brain. These findings demonstrate the clinical impact potential of RJX as a neuroprotective COVID-19 and sepsis drug candidate, which is currently being evaluated in a placebo-controlled, double-blind Phase II multiinstitutional US study in hospitalized patients with COVID-19 associated viral sepsis.

\section{Introduction}

Severe viral sepsis caused by SARS-CoV-2, the causative agent of coronavirus disease 2019 (COVID-19), shows a rapid progression associated with a cytokine release syndrome (CRS) and a high case fatality rate due to the development of ARDS and multi-organ failure in high-risk COVID-19 patients [1,2]. Our anti-sepsis drug candidate, Rejuveinix (RJX), is a rationally designed formulation of naturally occurring antioxidants and anti-inflammatory compounds with a significant clinical impact potential for COVID-19 associated viral sepsis $[3,4]$. RJX showed a very favorable clinical safety profile in a recently completed double-blind, placebo-controlled, randomized, two-part, ascending dose-escalation Phase 1 study in healthy volunteers (Protocol No. RPI003; ClinicalTrials.gov Identifier: NCT03680105) [5]. It has now entered a randomized, double-blind, placebo-controlled Phase 2 study in hospitalized patients with critical COVID-19.

More than a third of patients with COVID-19, especially those with severe to critical COVID-19 who are treated on an intensive care unit (ICU), develop central nervous system (CNS) symptoms and signs (e.g., headache, dizziness, ataxia, seizure, delirium, confusion, impaired consciousness) consistent with CNS involvement and /or neurological complications [6-13]. Cerebrovascular complications of viral sepsis, including ischemic or hemorrhagic stroke, CNS involvement in CRS, hypoxia as well as the interplay of comorbidities, have been implicated as contributing factors [6-13]. Due to the neuro-invasive capability of SARS-CoV-2, acute disseminated encephalomyelitis (ADEM) and viral encephalitis have also been suspected in some patients [13].

Oxidative stress caused by the massive production of reactive oxygen species (ROS) is thought to play a major role in the pathogenesis of severe viral sepsis in COVID-19 [14]. Chaudry et al. recently proposed that reactive oxygen intermediates and oxidative stress may play an important role in the pathophysiology of COVID-19 associated CNS disease, reminiscent of their role in Parkinson's disease [15]. Notably, RJX exhibited potent antioxidant activity and mitigated lipid peroxidation in prophylactic 
and therapeutic settings, as reflected by significantly decreased tissue MDA levels and normalization of the tissue levels of the antioxidant enzymes SOD, CAT, and GSH-Px as well as ascorbic acid [5]. The primary objective of the present study was to obtain experimental proof of concept that RJX can mitigate sepsis-associated oxidative stress in the brain.

\section{Materials And Methods}

\section{Rejuveinix (RJX)}

RJX is a proprietary composition of naturally occurring antioxidants and anti-inflammatory agents, which, in combination, provide potent and immediate tissue protection. Its ingredients include ascorbic acid, magnesium sulfate heptahydrate, cyanocobalamin, thiamine hydrochloride, riboflavin 5 ' phosphate, niacinamide, pyridoxine hydrochloride, and calcium D-pantothenate. RJX is a two-vial system, and A and $B$ are each of the two vials. Vial A contains the active ingredients and minerals, whereas Vial $B$ contains the buffer, sodium bicarbonate, as the Vial A content is acidic [5]. RJX is being developed as an antiinflammatory and antioxidant treatment platform for patients with sepsis, including COVID-19 patients with viral sepsis and ARDS [5].

\section{LPS-GaIN Model of Fatal Cytokine Storm, Sepsis, and Multi- organ Failure}

The ability of RJX, DEX, and RJX plus DEX to prevent fatal shock, ARDS, and multi-organ failure was examined in the well-established LPS-GalN model [5]. In this model, LPS is combined with GaIN, which further sensitizes mice to LPS-induced systemic inflammatory syndrome and multi-organ failure. Male BALB/c mice (6-8 weeks old) were obtained from the Firat University Experimental Animal Center. Mice had ad libitum access to standard rodent chow and water throughout the study. The care and treatment of the animals were in accordance with the Guide for the Care and Use of Laboratory Animals. The research protocol was approved by the Animal Care and Use Committee of Firat University. BALB/c mice were randomly divided into different treatment groups. All mice were genetically identical, of the same age, and the LPS-GalN challenged mice were injected with the same amount of LPS-GalN. This statistical equivalency of mice allowed using a pseudo-randomization convenience allocation to assign mice to identified cages. For random treatment allocation, cages were randomly selected to receive one of the specified treatments. We applied the concealment of treatment allocation and blind outcome assessment to reduce the risk of bias in our conclusions. Health care assessments were performed by animal care technicians not involved in treatment assignments or treatments. Investigators did not participate in individual health status or outcome assessments. Untreated normal control mice did not receive any treatments.

All mice except for the untreated normal control mice were challenged with an otherwise lethal dose of LPS mixed with GalN. Specifically, all mice were challenged with an i.p. injection of LPS plus Dgalactosamine (Sigma, St. Louis, MO). D-Galactosamine (Sigma Chemicals), which was dissolved at a 32 
$\mathrm{mg} / \mathrm{ml}$ final concentration in phosphate-buffered saline (PBS), was mixed with an equal volume of diluted, sonicated LPS immediately before dosing. This freshly prepared LPS-galactosamine mixture (LPS-GalN) was used immediately after preparation. Each mouse received a $500 \mu \mathrm{L}$ i.p. injection of LPSGalN (consisting of $100 \mathrm{ng}$ of LPS plus $8 \mathrm{mg}$ of D-galactosamine). In Part A of the study, vehicle control mice were treated with $0.5 \mathrm{~mL}$ normal saline (NS), i.e., an aqueous solution of $0.9 \% \mathrm{NaCl}$ instead of RJX. NS was administered intraperitoneally (i.p) 2 hours before and 2 hours after the i.p injection of LPS-GalN. Test mice received $0.7 \mathrm{~mL} / \mathrm{kg}$ RJX dose (2 hours before and 2 hours after LPS-GalN. Mice were monitored for mortality for $24 \mathrm{~h}$. The Kaplan-Meier method, log-rank chi-square test, was used to analyze the $24 \mathrm{~h}$ survival outcomes of mice in the different treatment groups. At the time of death, lungs and liver were harvested, fixed in $10 \%$ buffered formalin, and processed for histopathologic examination. $3 \mu \mathrm{m}$ sections were cut, deparaffinized, dehydrated, and stained with hematoxylin and eosin ( $\mathrm{H} \& \mathrm{E})$ and examined with light microscopy.

Lipid peroxidation as a biomarker of oxidative stress was determined and expressed as the amount of malondialdehyde (MDA, nmol/g tissue) in the brain, as previously described [5]. The enzymatic activities of SOD, CAT, and GSH-Px in the brain specimens were determined using the commercially available kits (Cayman Chemical, Ann Arbor, MI, USA) according to the manufacturer's procedures. In MDA assays, tissue samples $(0.3 \mathrm{~g})$ were analyzed for MDA using high-performance liquid chromatography (HPLC, Shimadzu, Kyoto, Japan) [5]. Specifically, an HPLC system equipped with the LC solution Software (Shimadzu, Kyoto, Japan), a pump (LC-20AD), a UV Detector (SPD-20A), a column oven (CTO-10ASVP), an autosampler (SIL-20 A), a degasser unit (DGU-20A5), and a column (Inertsil ODS-3, 250x 46 mm, 5 $\mathrm{mm}$ ) was used. Tissue samples were homogenized on ice in a glass-glass homogenizer in a mixture of $0.5 \mathrm{ml}$ of $\mathrm{HClO} 4(0.5 \mathrm{M}), 2.5 \mathrm{ml}$ distilled water, and 2[6]-di-tert-butyl-p-cresol (BHT). Then, the samples were centrifuged at $4500 \mathrm{rpm}$ for $5 \mathrm{~min}$, and supernatants were injected into the HPLC system. The addition of acid was necessary to precipitate proteins and release the MDA bound to the amino groups of proteins and other amino compounds. The mobile phase was $30 \mathrm{mM} \mathrm{KH} 2 \mathrm{PO} 4-$ methanol $(82.5+17.5$, $\mathrm{v} / \mathrm{v} \%, \mathrm{pH} 3.6$ ), and the flow rate was $1 \mathrm{ml} \mathrm{min}^{-1}$. The injection volume was $30 \mu \mathrm{L}$, and chromatograms were scanned at $250 \mathrm{~nm}$.

\section{Statistical Analyses}

Statistical analyses employed standard methods, including analysis of variance (ANOVA) and/or, nonparametric analysis of variance (Kruskal-Wallis) using the SPSS statistical program (IBM, SPPS Version 21), as reported [5]. Furthermore, the Kaplan-Meier method, log-rank chi-square test, was used to investigate survival and fatality in each group. P-values $<0.05$ were considered significant.

\section{Results}

\section{RJX improves survival outcomes after LPS-GalN induced sepsis.}

One hundred (100) percent (\%) of untreated control mice remained alive throughout the experiment. By comparison, $100 \%$ of LPS-GalN injected mice died at a median of 4.6 hours (Fig. 1). RJX was examined 
for its protective activity at a dose level, which is $>10$-fold lower than its maximum tolerated dose (MTD) of $0.759 \mathrm{~mL} / \mathrm{kg}$ for human subjects (viz.; $4.2 \mathrm{~mL} / \mathrm{kg}$ of a 6-fold diluted solution). RJX-treated mice had an improved survival outcome after being injected with LPS-GalN. In contrast to the invariably fatal treatment outcome of vehicle-treated control mice, $40 \%$ of mice treated with RJX $(n=25)$ remained alive with a 2.4-fold longer median time survival time of 10.9 hours (Log-rank $\left.X^{2}=20.60, P<0.0001\right)$ (Fig. 1).

\section{RJX reduces the oxidative stress in the brain after LPS-GaIN induced sepsis.}

No histopathologic brain lesions were observed in any of the mice challenged with LPS-GalN. However, the brain MDA levels measuring lipid peroxidation were markedly elevated, and the levels of the antioxidant enzymes SOD, CAT, and GSH-Px in the brain were dramatically reduced in LPS-GalN treated mice consistent with severe oxidative stress (Fig. 2). RJX decreased the brain MDA levels and normalized in a dose-dependent manner the reduced levels of the antioxidant enzymes SOD, CAT, and GSH-PX.

\section{Discussion}

COVID-19 has caused a marked increase in all-cause deaths in the US and has become the third leading cause of death for persons aged 45 through 84 years and the second leading cause of death for older persons. Patients with high-risk COVID-19 are in urgent need of treatment platforms capable of preventing the disease progression and/or reducing the case mortality rate by stopping or reversing the pulmonary as well as the systemic inflammatory process that causes the ARDS and culminates in multiorgan failure $[1,2,16]$. Here we extend our previous study and provide experimental evidence that RJX can improve the survival outcome in the LPS-GalN mouse model of fatal sepsis.

SOD, CAT, and GSH-Px are three pivotal antioxidant defense enzymes, and their levels are altered by the level of oxidative stress that is a hallmark of severe inflammation of sepsis [14]. Oxidative stress caused by the massive production of reactive oxygen species (ROS) is thought to play a major role in the pathogenesis of severe viral sepsis in COVID-19 as well [14]. The MDA levels were markedly elevated in the brain specimens of the LPS-GaIN challenged mice which is consistent with increased lipid peroxidation. In parallel, the levels of the antioxidant enzymes SOD, CAT, and GSH-Px were profoundly suppressed due to severe oxidative stress. RJX exhibited potent antioxidant activity and mitigated lipid peroxidation, as reflected by significantly decreased tissue MDA levels and normalization of the tissue levels of the antioxidant enzymes SOD, CAT, and GSH-Px as well as ascorbic acid. We hypothesize that RJX will shorten the time to resolution of ARDS and viral sepsis in COVID-19 patients by preventing the development of a fulminant cytokine storm as well as reversing the cytokine-mediated multi-system inflammatory process and oxidative stress, thereby mitigating the inflammatory organ injury.

Oxidative stress owing to mitochondrial dysfunction has been implicated in the pathophysiology of Alzheimer's disease (AD), the most common form of dementia, Parkinson's disease (PD), the second most common progressive disorder of the central nervous system, and Huntington's disease (HD), a neurodegenerative disorder associated with cognitive decline and dementia. Notably, in a mouse CNS 
model of severe oxidative stress, RJX rapidly and substantially increased the levels of the antioxidant enzymes SOD, CAT, GSH-Px that were reduced in the brains of LPS-GalN treated mice consistent with the severe oxidative stress. The results presented herein demonstrate for the first time that RJX could have therapeutic utility in the treatment of $A D, P D, H D$, and ALS. Furthermore, because of the well-established role of oxidative stress in the development and progression of ischemic stroke, one of the leading causes of mortality and morbidity, RJX could significantly improve the standard of care for stroke as well.

\section{Declarations}

\section{Acknowledgments}

The authors thank Reven Pharmaceuticals, LLC (Westminster, CO USA) for supporting the project.

\section{Authors' contributions}

Each author has made significant and substantive contributions to the study, reviewed and revised the manuscript, provided final approval for submitting the final version. No medical writer was involved. F.M.U conceived the study, designed the evaluations reported in this paper, directed the data compilation and analysis, analyzed the data, and prepared the initial draft of the manuscript. Each author had access to the source data used in the analyses.

\section{Funding}

This study was funded by Reven Pharmaceuticals, LLC, a wholly-owned subsidiary of Reven Holdings Inc

\section{Availability of data and material}

Sets of data or summaries generated during the present study are available from the corresponding author upon reasonable request.

\section{Ethics approval}

The care and treatment of the animals were in accordance with the Guide for the Care and Use of Laboratory Animals. The research protocol was approved by the Animal Care and Use Committee of Firat University.

\section{Consent to participate}

Informed consent was obtained from all individual participants included in the current study.

\section{Consent for publication}

Consent of publication was obtained from all authors

\section{Conflicts of interest}


F.M.U. and M.V are employees of Reven Pharmaceuticals, the sponsor for the clinical development of RJX. M.T., M.G, and K.S declare no current competing financial interests

\section{References}

1. Uckun FM (2020) Reducing the fatality rate of Covid-19 by applying clinical insights from immunooncology and lung transplantation. Front Pharmacol 11:796. doi:10.3389/fphar.2020.00796

2. Jiang F, Deng L, Zhang L, Cai Y, Cheung CW, Xia Z (2020) Review of the clinical characteristics of coronavirus disease 2019 (COVID-19). J Gen Intern Med 35(5):1545-1549. doi:10.1007/s11606-02005762-w

3. Cabala K, Earabino J, Pacult P, Uckun FM (2020) Rationale for a randomized, placebo-controlled, phase 2 study of Rejuveinix (RJX) in COVID-19 patients with acute lung injury and hypoxemic respiratory failure. J Clin Invest 10(2):185-189

4. Uckun FM, Ervin J, Sahin K, Powell J, Pizzimenti N, Earabino J, Van Wyk H, Van Wyk M, Pacult P, Sahin TK, Cabala K (2020) Clinical impact potential of Rejuveinix (RJX) for prevention of fatal acute respiratory distress syndrome (ARDS) and multi-organ failure in Covid-19 patients. J Clin Invest 10(2):177-184. doi:10.37532/2041-6792.2020.10(2).167

5. Uckun FM, Carlson J, Orhan C, Powell J, Pizzimenti NM, Van Wyk H, Ozercan IH, Volk M, Sahin K (2020) Rejuveinix shows a favorable clinical safety profile in human subjects and exhibits potent preclinical protective activity in the Lipopolysaccharide-Galactosamine mouse model of acute respiratory distress syndrome and multi-organ failure. Front Pharmacol 11:594321. doi:10.3389/fphar.2020.594321

6. Azhideh A (2020) Covid-19 neurological manifestations. Int Clin Neurosci J 7(2):54. doi:10.34172/icnj.2020.01

7. Soltani Zangbar H, Gorji A, Ghadiri T (2021) A review on the neurological manifestations of Covid-19 infection: a mechanistic view. Mol Neurobiol 58(2):536-549. doi:10.1007/s12035-020-02149-0

8. Wu Y, Xu X, Chen Z, Duan J, Hashimoto K, Yang L, Liu C, Yang C (2020) Nervous system involvement after infection with Covid-19 and other coronaviruses. Brain Behav Immun 87:18-22. doi:10.1016/j.bbi.2020.03.031

9. Achar A, Ghosh C (2020) Covid-19-associated neurological disorders: the potential route of CNS invasion and blood-brain relevance. Cells 9(11):2360. doi:10.3390/cells9112360

10. Verma K, Amitabh, Prasad DN, Kumar B, Kohli E (2020) brain and Covid-19 crosstalk: pathophysiological and psychological manifestations. ACS Chem Neurosci 11(20):3194-3203. doi:10.1021/acschemneuro.0c00446

11. Alharthy A, Faqihi F, Memish ZA, Karakitsos D (2020) Fragile endothelium and brain dysregulated neurochemical activity in Covid-19. ACS Chem Neurosci 11(15):2159-2162. doi:10.1021/acschemneuro.0c00437 
12. Niazkar HR, Zibaee B, Nasimi A, Bahri N (2020) The neurological manifestations of Covid-19: a review article. Neurol Sci 41(7):1667-1671. doi:10.1007/s10072-020-04486-3

13. Hassanzadeh K, Perez Pena H, Dragotto J, Buccarello L, lorio F, Pieraccini S, Sancini G, Feligioni M (2020) Considerations around the SARS-CoV-2 spike protein with particular attention to COVID-19 brain infection and neurological symptoms. ACS Chem Neurosci 11(15):2361-2369. doi:10.1021/acschemneuro.0c00373

14. Schönrich G, Raftery MJ, Samstag Y (2020) Devilishly radical NETwork in COVID-19: Oxidative stress, neutrophil extracellular traps (NETs), and T cell suppression. Adv Biol Regul 77:100741. doi:10.1016/j.jbior.2020.100741

15. Chaudhry ZL, Klenja D, Janjua N, Cami-Kobeci G, Ahmed BY (2020) COVID-19 and Parkinson's disease: shared inflammatory pathways under oxidative stress. Brain Sci 10(11):807. doi:10.3390/brainsci10110807

16. Zumla A, Hui DS, Azhar El, Memish ZA, Maeurer M (2020) Reducing mortality from 2019-nCoV: hostdirected therapies should be an option. Lancet 395(10224):e35-e36. doi:10.1016/S01406736(20)30305-6

\section{Figures}

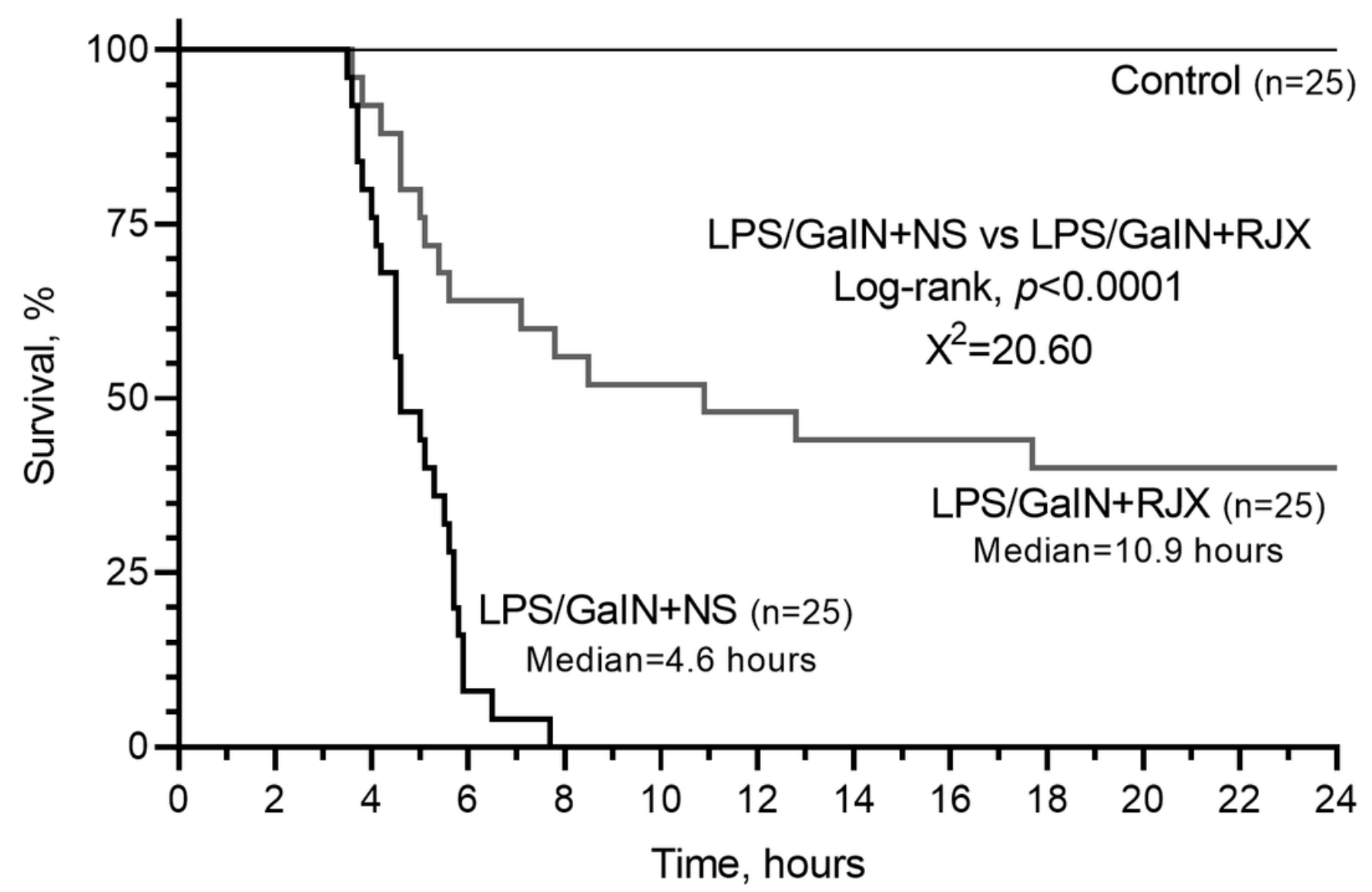


Figure 1

In Vivo Protective Activity of RJX in the LPS-GALN challenged mice. Groups of 25 BALB/C mice were treated with i.p injections of 6-fold diluted RJX ( $4.2 \mathrm{~mL} / \mathrm{kg}, 0.5 \mathrm{ml} / \mathrm{mouse})$ or vehicle (NS) 2 hours before or post-injection of LPS-GaIN. Except for untreated mice (Control), each mouse received $0.5 \mathrm{ml}$ of LPSGalN (consisting of $100 \mathrm{ng}$ of LPS plus $8 \mathrm{mg}$ of D-galactosamine) i.p. Survival is shown as a function of time after the LPS-GalN challenge. Depicted are the survival curves for each group along with the median survival times and the log-rank P-value for the comparison of the LPS-GalN+RJX group versus the LPS/GaIN+NS group.
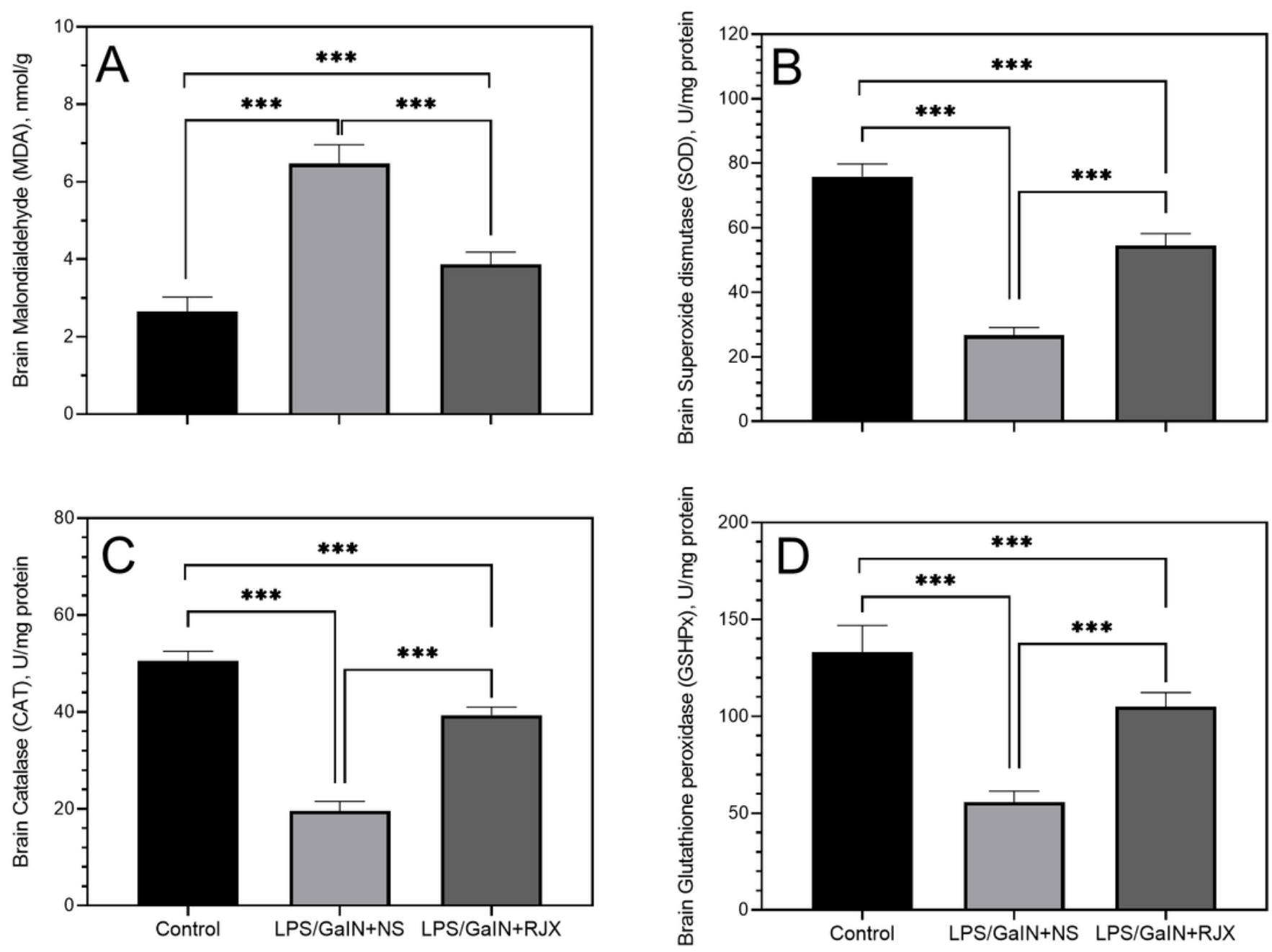

Figure 2

Effect of Rejuveinix (RJX) on brain malondialdehyde (MDA; Panel A), superoxide dismutase (SOD; Panel B), catalase (CAT; Panel C), and Glutathione peroxidase (GSHPx; Panel D) in the lipopolysaccharidegalactosamine (LPS-GaIN) challenged mice. Each bar represents the mean and standard deviation for the measured parameter of mice from each specific treatment group. BALB/C mice were treated with i.p injections of 6-fold diluted RJX (4.2 mL/kg, $0.5 \mathrm{ml} / \mathrm{mouse})$ or vehicle (NS) 2 hours before or post-injection 
of LPS-GalN. Except for untreated mice (Control), each mouse received $0.5 \mathrm{ml}$ of LPS-GalN (consisting of $100 \mathrm{ng}$ of LPS plus $8 \mathrm{mg}$ of D-galactosamine) i.p. (ANOVA and Tukey's post-hoc test. Statistical significance between groups is shown by: $\left.{ }^{\star \star \star} P<0.001\right)$. 